\title{
VIVÊNCIA DOS TRABALHADORES RURAIS FRENTE AO USO DE AGROQUÍMICOS: UMA REVISÃO INTEGRATIVA
}

\author{
EXPERIENCE OF RURAL WORKERS IN FRONT OF THE USE OF \\ AGROCHEMISTS: AN INTEGRATIVE REVIEW
}

Laura Aires Cavalcante Leite ${ }^{1}$

Renata Ferreira de Araújo ${ }^{2}$

Anny Isabelly Medeiros de Góes ${ }^{3}$

Karén Kelyany Duarte Costa ${ }^{4}$

Francilene Maciel Ferreira da Silva ${ }^{5}$

\begin{abstract}
RESUMO: O Brasil é um dos maiores consumidores mundiais dos agroquímicos. Devido a esse fato, encontra-se susceptível a maiores índices de intoxicação. Estes, por sua vez, caracterizam-se por um conjunto de efeitos nocivos, desencadeando o desequilíbrio do organismo dos trabalhadores, por meio de um ou mais agentes tóxicos. Desse modo, o objetivo centra-se nas dificuldades enfrentadas pelos trabalhadores rurais, frente ao uso dos agroquímicos. Para isso, foi feito uma revisão integrativa, realizada por intermédio da Biblioteca Virtual em Saúde (BVS). A revisão considerou a compilação de artigos mais recentes, entre 2016 e 2020, centrados na temática dos agroquímicos e dos trabalhadores que fazem uso deles. Como resultado, foram selecionados seis artigos, que mostraram sobretudo a predominância de trabalhadores rurais que fazem uso de agentes tóxicos como sendo sexo masculino, com idadesentre 30 e 60 anos, renda menor ou igual a dois salários-mínimos. Dentre as principais dificuldades enfrentadas estão o uso de EPI, consequências na saúde física e emocional, despreparo com relação ao uso dos agroquímicos e falta de receita agronômica. Desta feita, observa-se que os trabalhadores rurais representam um grupo que necessita de cuidado e monitoramento, para avaliar e amenizar as consequências do uso de agroquímicos no cotidianodos mesmos.
\end{abstract}

Palavras chave: Agroquímicos. Saúde da População Rural. Trabalhadores Rurais.

\footnotetext{
${ }_{1}^{1}$ Graduanda em Enfermagem pela Universidade Estadual da Paraíba;

${ }^{2}$ Graduanda em Enfermagem pela Universidade Estadual da Paraíba e bolsista de IniciaçãoCientífica CNPq, UEPB.

${ }^{3}$ Graduanda em Enfermagem pela Universidade Estadual da Paraíba e bolsista de IniciaçãoCientífica CNPq, UEPB;

${ }^{4}$ Graduanda em Enfermagem pela Universidade Estadual da Paraíba;

${ }^{5}$ Graduanda em Enfermagem pela Universidade Estadual da Paraíba.
} 
ABSTRACT: Brazil is one of the world's largest consumers of agrochemicals. Due to this fact, it is susceptible to higher rates of intoxication. These, in turn, are characterized by a set of harmful effects, triggering the imbalance of the workers' organism, through one or more toxic agents. Thus, the objective is centered on the difficulties faced by rural workers, regarding the use of agrochemicals. For this, an integrative review was carried out, carried out through the Virtual Health Library (VHL). The review considered the compilation of more recent articles, between 2016 and 2020, focused on the theme of agrochemicals and the workers who use them. As a result, six articles were selected, which showed above all the predominance of rural workers who use toxic agents as being male, aged between 30 and 60 years, income less than or equal to two minimum wages. Among the main difficulties faced are the use of PPE, consequences on physical and emotional health, unpreparedness in relation to the use of agrochemicals and lack of agronomic prescription. This time, it is observed that rural workers represent a group that needs care and monitoring, to assess and mitigate the consequences of using agrochemicals in their daily lives.

Keywords: Agrochemicals. Rural Health. Rural Workers. 


\title{
INTRODUÇÃO
}

A problematização dos diversos tipos de agrotóxicos gira em torno de vários âmbitos: sociais, ambientais e econômicos. Os problemas ambientais discutem sobre o impactono ecossistema como um todo, a exemplo da contaminação do ar, da água, da terra e dos animais que são atingidos e que apresentam grau de toxicidade. Os impactos econômicos abarcam a responsabilização do Estado por toda a problemática gerada pelas intoxicações por agrotóxicos, visto que as despesas são arcadas pelo Estado e não pelas empresas produtoras de agrotóxicos. No âmbito social, os agrotóxicos têm gerado discussões acerca do conjunto da sociedade, sendo a mais destacada a saúde da população (FOLGADO, 2014).

A legislação brasileira traz que:

\begin{abstract}
"Agrotóxicos são produtos e agentes de processos físicos, químicos ou biológicos, destinados ao uso nos setores de produção, no armazenamento e beneficiamento de produtos agrícolas, nas pastagens, na proteção de florestas, nativas ou implantadas, e deoutros ecossistemas e também de ambientes urbanos, hídricos e industriais, cuja finalidade seja alterar a composição da flora ou da fauna, a fim de preservá-las da ação danosa de seres vivos considerados nocivos, bem como as substâncias e produtos empregados como desfolhantes, dessecantes, estimuladores e inibidores de crescimento." (BRASIL, 1989, p. 11459)
\end{abstract}

Os principais grupos de agrotóxicos, segundo o uso, são os agrotóxicos de uso agrícola, agrotóxico de uso doméstico, agrotóxico de uso em saúde pública e raticidas e alguns produtos veterinários. O Brasil é um dos maiores consumidores mundiais dos agrotóxicos, sendo, desta forma, susceptível a maiores índices de intoxicação, que se caracteriza pelo conjunto de efeitos nocivos que revelam o desequilíbrio do organismo produzida pela interação de um ou mais agentes tóxicos (BRASIL, 2018; BRASIL, 2016).

Abrasco (2015) relata que um terço dos alimentos consumidos cotidianamente está contaminado pelos agrotóxicos, segundo análise de amostras 
coletadas em todos os 26 estados do Brasil através do Programa de Análises de Resíduos de Agrotóxicos em Alimentos (PARA) da Anvisa (2011). Aumentando, dessa forma, a insegurança alimentar da população, tendo emvista que não se sabe ao certo quanto de agrotóxico é ingerido, perdendo o limiar de toxicidade.

As intoxicações por agrotóxicos se dividem em agudas e crônicas, sendo intoxicações agudas aquelas que apresentam repercussão imediata, logo após o contato com o agrotóxico. Ea crônica acontece quando os sintomas acontecem de forma tardia, apresentando meses ou anosapós o contato com o agente causador. As intoxicações crônicas são as que mais necessitam de investigação, pois, muitas vezes os sintomas são facilmente associados a outro agente causador (ABRASCO, 2015).

É sabido que os profissionais de saúde enfrentam no Brasil enormes dificuldades para diagnosticar, registrar e direcionar os pacientes com sintomas de intoxicação por agrotóxico. Além disso, o número de registros é menor do que o número real por intoxicações, fato que a própria Organização Mundial de Saúde reconhece que para cada caso registrado de intoxicação, cinquenta não são notificados. Ademais, as pessoas que trabalham no campo são as que mais correm risco de contaminação por agrotóxicos. Alguns exemplos de grupo de risco são os moradores de regiões de agronegócio, os aplicadores que tenham contato direto, trabalhadores que realizam capinas, roçados ou colheita, os profissionais de saúde que trabalham frente as arboviroses, os funcionários de empresas de dedetização, funcionários que fabricam ou formulam agrotóxicos, bem como quem trabalha com o transporte e o comércio desses materiais(LONDRES, 2011).

Assim como sugere Cezar-Vaz, Bonow, Mello e Silva (2016) no seu estudo, a associação entre o trabalho rural e o uso de agrotóxicos permite expandir acessos para a construção do conhecimento de Enfermagem. A partir disso, supõe-se que o presente estudo vai contribuir de forma positiva para a área da Enfermagem em saúde pública, no que diz respeito ao cuidado com a população de trabalhadores rurais expostos a agrotóxicos, trazendo associação teórica para embasar a prática do profissional de enfermagem. O objetivo desse estudo é identificar quais são as dificuldades enfrentadas pelos trabalhadores rurais frente ao uso de agrotóxicos. 


\section{MATERIAIS E MÉTODOS}

Trata-se de uma revisão integrativa da literatura, em que busca sintetizar as pesquisas disponíveis sobre determinada temática e direcionar a prática fundamentando-se em conhecimento científico. Para a sua elaboração, utilizou-se seis fases: 1) Elaboração da pergunta norteadora; 2) Busca ou amostragem na literatura; 3) Coleta de dados; 4) Análise crítica dos estudos incluídos; 5) Discussão dos resultados; 6) Apresentação da revisão integrativa (SOUZA; SILVA; CARVALHO, 2010).

A revisão teve como pergunta norteadora: Quais são as dificuldades enfrentadas pelos trabalhadores rurais frente ao uso de agrotóxicos? A busca dos artigos ocorreu no período de janeiro de 2021, na Biblioteca Virtual em Saúde (BVS). Os Descritores em Ciência da Saúde (DECS) utilizados como termos foram: "Agroquímicos", "Saúde da População Rural" e "Trabalhadores Rurais". Os cruzamentos foram realizados utilizando o operador booleano (AND), com os seguintes cruzamentos: "Agroquímicos" AND "Saúde da População Rural" e "Agroquímicos" AND "Trabalhadores Rurais". O resultado dos artigos encontrados por cada cruzamento está explanado mais detalhado na figura 1.

Os critérios de inclusão foram: artigos originais disponíveis na íntegra, com texto completo, no idioma português, artigos com ano de publicação maior que 2015 e que respondessem à pergunta norteadora. Já os critérios de exclusão foram: artigos incompletos, teses, dissertações, artigos com ano de publicação menor que 2015 e que não respondessem à pergunta norteadora. 


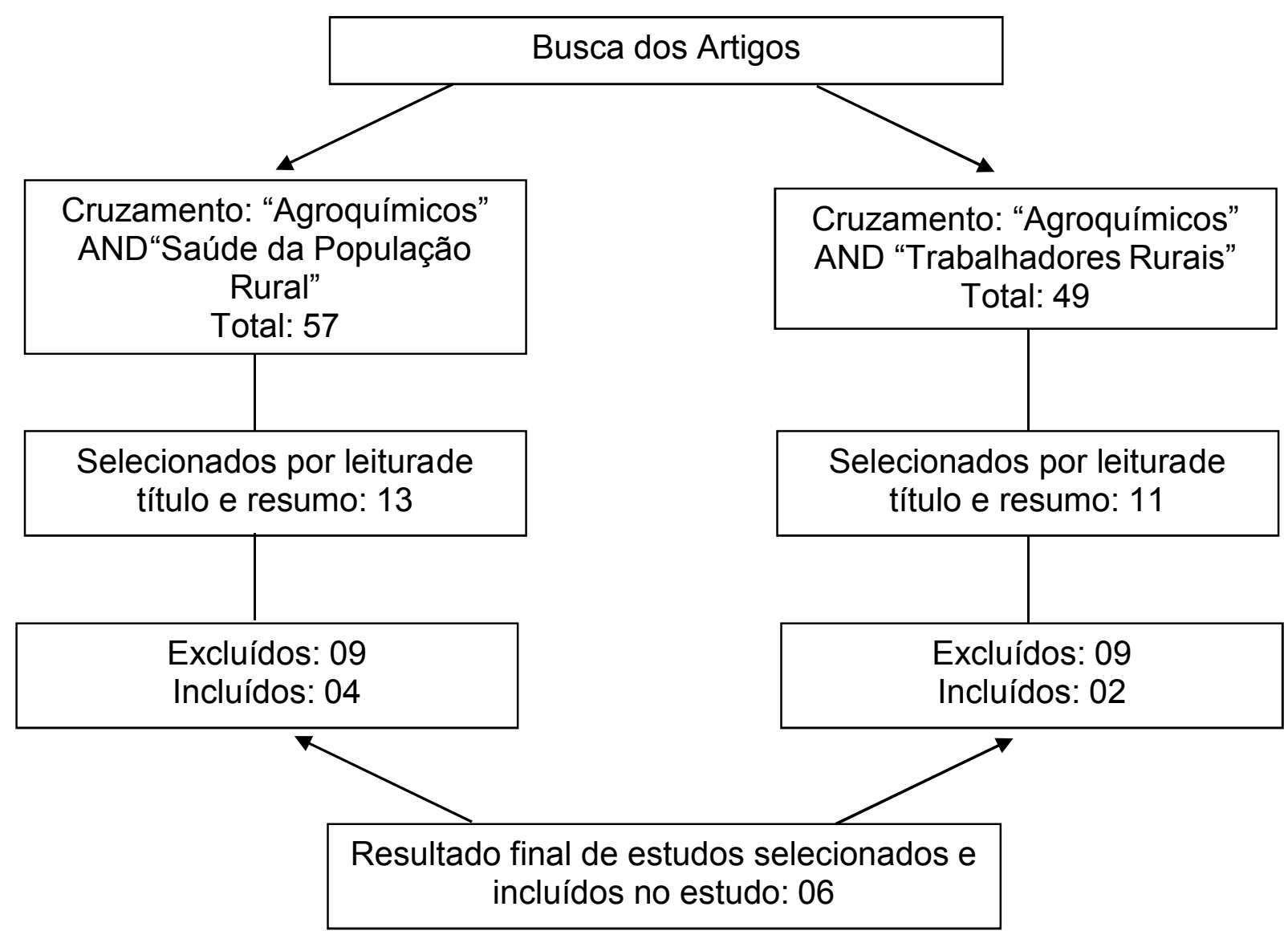

Figura 1. Fluxograma da pesquisa e seleção dos artigos para a revisão integrativa da literatura, 2021.

Após análise dos dados, foram incluídos nesta revisão integrativa da literatura um total de 06 artigos, tendo suas descrições abordadas no Quadro 1. Os artigos incluídos na revisão são todos do idioma português (100\%). Sendo o ano de 2020 com 1 artigo (16,67\%), 1 artigo de 2019 (16,67\%), 2 artigos de 2018 (33,33\%) e 2 artigos de $2016(33,33 \%)$. 
Quadro 1 - Descrição dos artigos incluídos na revisão integrativa, segundo autores, ano depublicação, título, periódico, delineamento e objetivo do estudo.

\begin{tabular}{|c|c|c|c|c|}
\hline $\begin{array}{l}\text { Autores/Ano } \\
\text { dePublicação }\end{array}$ & Título & Periódico & $\begin{array}{c}\text { Delineamentodo } \\
\text { Estudo }\end{array}$ & Objetivo doEstudo \\
\hline $\begin{array}{l}\text { RISTOW; } \\
\text { BATTISTI; } \\
\text { STUMM; } \\
\text { MONTAGNER, } \\
2020\end{array}$ & $\begin{array}{l}\text { Fatores } \\
\text { relacionados à } \\
\text { saúde } \\
\text { ocupacional de } \\
\text { agricultores } \\
\text { expostos } \\
\text { agrotóxicos. }\end{array}$ & $\begin{array}{l}\text { Revista } \\
\text { Saúde e } \\
\text { Sociedade }\end{array}$ & $\begin{array}{l}\text { Tran } \\
\text { obse }\end{array}$ & $\begin{array}{l}\text { Analisar se } \\
\text { características } \\
\text { sociodemográficas, } \\
\text { eapacitação técnica e } \\
\text { percepção de risco } \\
\text { estão relacionados com } \\
\text { a saúde ocupacional de } \\
\text { agricultores Expostos a } \\
\text { agrotóxicos }\end{array}$ \\
\hline $\begin{array}{l}\text { CO } \\
\text { al., }\end{array}$ & $\begin{array}{l}\text { Avaliação do } \\
\text { efeito do uso } \\
\text { de agrotóxicos } \\
\text { sobre a saúde } \\
\text { de } \\
\text { trabalhadores } \\
\text { rurais } \\
\text { fruticultura da } \\
\text { irrigada. }\end{array}$ & $\begin{array}{l}\text { Revista } \\
\text { Ciência } \\
\text { Saúde } \\
\text { Coletiva }\end{array}$ & $\begin{array}{l}\text { Descritivo, } \\
\text { abordagem } \\
\text { quantitativa }\end{array}$ & 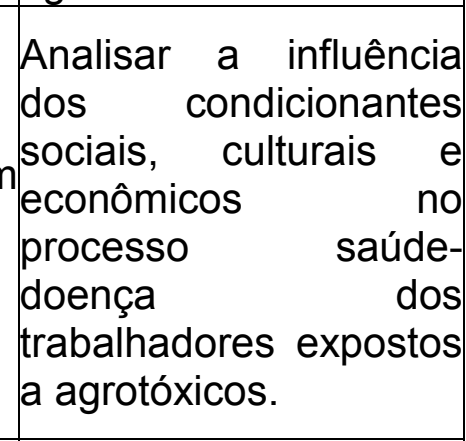 \\
\hline $\begin{array}{l}\text { MORIN; } \\
\text { STUMM, } 2018\end{array}$ & \begin{tabular}{|l|} 
Transtornos \\
mentais \\
comuns em \\
agricultores, \\
relação com \\
agrotóxicos, \\
sintomas \\
físicos \\
doenças \\
preexistentes
\end{tabular} & $\begin{array}{l}\text { Revista } \\
\text { Psico }\end{array}$ & $\begin{array}{l}\text { Transversal, } \\
\text { descritivo } \\
\text { analítico }\end{array}$ & 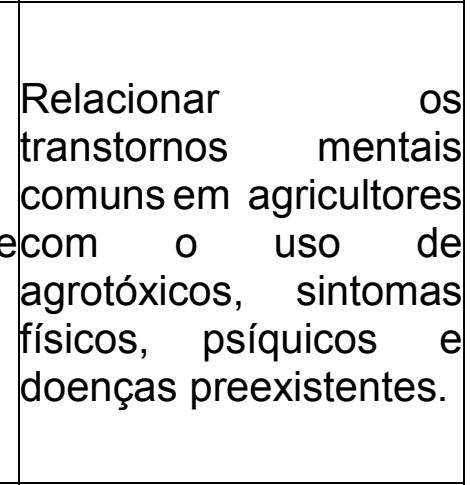 \\
\hline $\begin{array}{l}\text { FENZKE; } \\
\text { MELLO; } \\
\text { SANTOS; } \\
\text { CEZAR-VAZ, } \\
2018\end{array}$ & 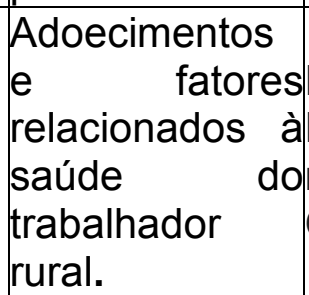 & $\begin{array}{l}\text { Revista de } \\
\text { Enfermage } \\
\text { m, UFPE } \\
\text { Online }\end{array}$ & a & $\begin{array}{l}\text { Identificar os fatores } \\
\text { que levam } \\
\text { adoecimento } \\
\text { trabalhador rural que } \\
\text { utiliza agrotóxico. }\end{array}$ \\
\hline $\begin{array}{l}\text { VIERO et al. } \\
2016\end{array}$ & $\begin{array}{l}\text { Sociedade re } \\
\text { risco: o uso dos } \\
\text { agrotóxicos r } \\
\text { implicações } \\
\text { saúde } \\
\text { trabalhador } \\
\text { rural. }\end{array}$ & $\begin{array}{l}\text { Revista de } \\
\text { Enfermage } \\
\text { m - Escola } \\
\text { Anna Nery }\end{array}$ & $\begin{array}{l}\text { ritivo, } \\
\text { dagem } \\
\text { tativa }\end{array}$ & $\begin{array}{llr}\text { Conhecer } & \text { as } \\
\text { percepções } & \text { de } \\
\text { ntrabalhadores } & \text { rurais } \\
\text { sobre os } & \text { riscos } \\
\text { advindos do uso de } \\
\text { agrotóxicos para sua } \\
\text { saúde. }\end{array}$ \\
\hline
\end{tabular}




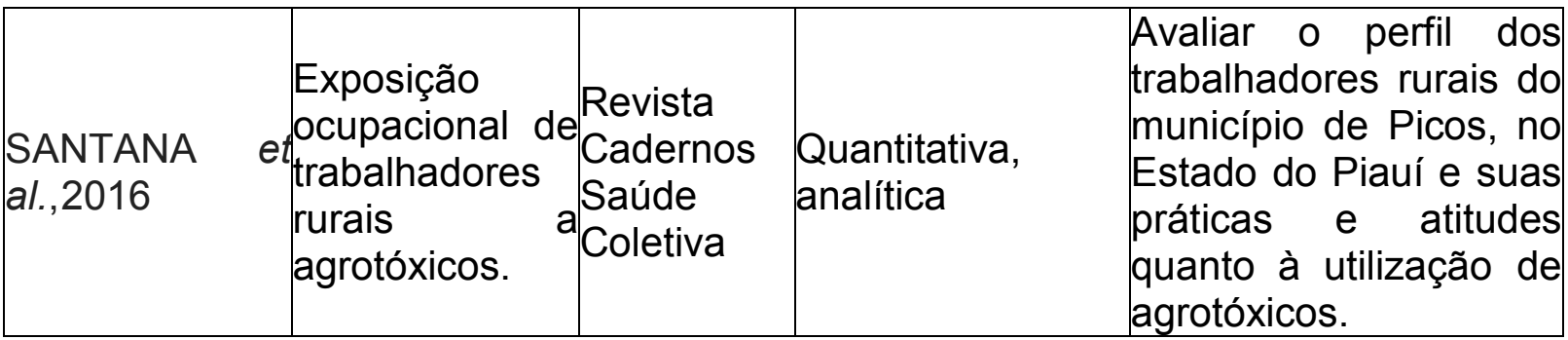

Fonte: Construção dos autores, 2021.

\section{RESULTADOS E DISCUSSÕES}

A rotina do trabalhador rural que utiliza agrotóxicos apresenta-se envolta de várias questões que dizem respeito não apenas ao trabalhador. $O$ treinamento quanto ao uso dos EPI's(Equipamentos de Proteção Individual), por exemplo, é uma das questões que são pouco abordadas ao trabalhador. Ristow, Battisti, Stumm e Montagner (2020) observou em sua pesquisa que os agricultores não possuem capacitação técnica por parte dos órgãos públicos, recebendo, assim, treinamento para uso dos agrotóxicos apenas por meio das empresas que comercializam o produto. Santana et al. (2016) conclui em sua pesquisa que os agricultores obtinham informações sobre o uso dos agrotóxicos através de vizinhos, liam o rótulo para utilizar o produto, comprovam em casas agropecuárias e guardavam as embalagens dentro da própria casa.

Corcino et al. (2019) e Santana et al. (2016) ainda acrescentaram que na sua pesquisa, os trabalhadores rurais sabiam que para a utilização dos agroquímicos necessitavam do uso de receituário agronômico, mas desconsideravam a informação. Além disso, ainda conhecem os riscos que estão sendo expostos quando não fazem o uso inadequado dos agroquímicos e dos EPl's. Na discussão que Viero et al. (2016) trouxeram com os participantes da sua pesquisa, dois deles acrescentam que tem o entendimento que várias patologias (a exemplo do câncer) está surgindo devido ao uso dos agroquímicos, e relata que quando era criança não lembra de encontrar casos como hoje se encontra facilmente.

Fenzke, Mello, Santos e Cezar-vaz (2018) constataram em sua revisão integrativa que $43,59 \%$ dos artigos traziam que a maioria dos agricultores não fazem 
uso dos EPI's. Em concordância com essa informação, Santana et al. (2016) revelou no seu estudo que $56,8 \%$ dos profissionais também não fazem uso dos EPI's no seu cotidiano. Ristow, Battisti, Stumm e Montagner (2020) conseguiram identificar algumas dúvidas dos profissionais relacionadas a práticas laborais, doenças ou infecções por agrotóxicos e produtos (dosagem, forma, aplicação). Dessa forma, percebe-se que uma das dificuldades encontradas é a falta de informação dos profissionais acerca das práticas realizadas pelos mesmos.

O tópico idade foi evidenciado em quase a totalidade dos artigos, sendo que Santana et al. (2016) mostrou que mais de $50 \%$ da sua amostra possuía entre 31 e 50 anos, Ristow, Battisti, Stumm e Montagner (2020) mostrou que 53\% tinham entre 51 a 76 anos, Morin e Stumm (2018) mostrou prevalência entre as faixas etárias de 40 a 60 anos incompletos, Viero et al. (2016) apresentou prevalência entre 41 e 60 anos. Diante dessas informações, Corcino et al. (2019) traz em seu estudo uma observância no que diz respeito a vulnerabilidade do fator idade, visto que jovens e idosos são mais susceptíveis ao adoecimento, citando o estudo realizado por Silvaet al. (2013) que evidenciou que sintomas como visão alterada, boca seca (xerostomia) e dor nas pernas foram mais prevalentes em idosos que fazem o uso de agrotóxicos.

Santana et al. (2016) constatou no seu estudo que o nível de escolaridade dos trabalhadores rurais $(55,6 \%)$ é de nível fundamental, fato que muitas vezes dificulta o entendimento com relação as condutas a serem tomadas com os EPI's e com os agrotóxicos. Morin e Stumm (2018) e Corcino et al. (2019) também avaliaram em suas pesquisas questões referentes ao nível de escolaridade, evidenciando que mais de $50 \%$ dos trabalhadores possuíam apenas ensino fundamental incompleto, reforçando, dessa forma, que é uma lacuna a ser fechada.

Outro ponto apontado por Santana et al. (2016) e Corcino et al. (2019) foi a renda dos trabalhadores participantes das pesquisas, em que $66 \%$ possuíam como renda até um salário para Santana et al. (2016) e que para Corcino et al. (2019) $55,2 \%$ dos participantes tinha rendimentos mensais menor ou igual a 2 salários mínimos. Dessa forma, percebe-se que o riscoé alto e o benefício financeiro é baixo para essa população, sendo este uma das dificuldades enfrentadas pelos trabalhadores rurais que utilizam agroquímicos. 
A saúde do trabalhador rural que faz uso de agrotóxicos torna-se cada vez mais prejudicada. Morin e Stumm (2018) traz que um dos sintomas mais prevalentes após a utilização dos agrotóxicos é dor de cabeça, irritação nos olhos, visão turva, lacrimejamento, lesões de pele, tontura, náuseas, vômito, aumento de saliva, suor excessivo, tosse, boca seca, chiado no peito, falta de ar, dor abdominal, digestão difícil, tremores, diarreia, cansaço físico, coceira na pele, gastrite, espirros e sente-se mal com inseticida. Outro aspecto discutido é de aspectos emocionais serem também atingidos, se fazendo presente sintomas como agitação, irritabilidade, insônia, desânimo, cansaço mental, tristeza e dificuldade de concentração.

Com relação aos sintomas crônicos, Ristow, Battisti, Stumm e Montagner (2020) associa enfisema pulmonar, câncer de pele, câncer no pâncreas, câncer na cavidade nasal, doença de Alzheimer, deficiência mental e problemas respiratórios. Fenzke, Mello, Santos e Cezar-vaz (2018) conseguiu mensurar em seu estudo que a maior ocorrência é em alterações do sistema nervoso (intoxicações, convulsões, doenças neurológicas, neuropatia periférica, doença de Parkinson e transtornos mentais/psiquiátricos). Em segundo lugar, estão as alterações sensoriais (irritação ocular, perda auditiva, síndrome vestibular periférica irritativa, perda auditiva sensorioneural e problemas no processamento auditivo temporal) e em terceiro lugar as alterações do sistema respiratório (rinite alérgica, asma e doença obstrutiva crônica).

\section{CONSIDERAÇÕES FINAIS}

As principais dificuldades vivenciadas pelos profissionais rurais que fazem uso de agrotóxicos destacadas neste estudo são o uso de EPI (Equipamento de Proteção Individual), e, que boa parte das pesquisas evidenciaram o não uso ou o uso inadequado dos mesmos, ressaltando importância de investimento de conhecimento nesta população. O fator idade mais prevalente foi entre 40-60 anos, mostrando que é uma faixa etária que apresenta fragilidades. O sexo mais 
prevalente foi o masculino e a renda média entre os participantes da pesquisa foi de um salário, podendo chegar a dois salários mínimos.

O uso de receituário agronômico apresentou-se ausente na maioria das amostras de trabalhadores estudadas pelas pesquisas, mostrando que o uso dos agroquímicos é feita de formaleiga e irregular, aumentando a chance de intoxicação aguda e crônica. Ainda assim, otrabalhador tem conhecimento dos riscos que estão sendo expostos, mostrando que a ocorrência de intoxicação crônica já é esperada por muitos deles. Além disso, os estudos mostraram consequências na saúde física e emocional dos profissionais, ressaltando a importância do monitoramento dessa população de trabalhadores.

\section{REFERÊNCIAS BIBLIOGRÁFICAS}

ABRASCO. Dossiê Abrasco: um alerta sobre os impactos dos agrotóxicos na saúde. Rio de Janeiro: Expressão Popular, 2015. 628p. Disponível em: https://www.arca.fiocruz.br/bitstream/icict/26221/2/Livro\%20EPSJV\%20013036.pdf. Acessoem: 1 fev. 2021.

BRASIL. Ministério da Saúde. Secretaria de Vigilância em Saúde. Coordenação-Geral de Desenvolvimento da Epidemiologia em Serviços. Guia de Vigilância em Saúde. 1a edição. Brasília: Ministério da Saúde; 2016. 775 p. Disponível em: http://bvsms.saude.gov.br/bvs/publicacoes/guia_vigilancia_saude_1ed_atual.pdf. Acesso em:20 fev. 2021.

BRASIL. Ministério da Saúde. Secretaria de Vigilância em Saúde. Departamento de Vigilância em Saúde Ambiental e Saúde do Trabalhador. O agente comunitário de saúde na prevenção das intoxicações por agrotóxicos. Brasília: Ministério da Saúde, 2018. 21p.

Disponível em: http://bvsms.saude.gov.br/bvs/publicacoes/agente_comunitario_saude_agrotoxicos.pdf. Acesso em: 20 fev. 2021.

BRASIL. Presidência Da República. Diário Oficial da União, de 11 de julho de 1989, Seção1, página 11459; 1989. Disponível em: https://www2.camara.leg.br/legin/fed/lei/1989/lei-7802-11julho-1989-356807-publicacaooriginal-1-pl.html. Acesso em: 20 fev. 2021.

CEZAR-VAZ, Marta Regina; BONOW, Clarice Alves; MELLO, Marlise Capa Verde Almeida de; SILVA, Mara Regina Santos da. Abordagem socioambiental na enfermagem: focalizando o trabalho rural e uso de agrotóxicos. Revista Brasileira de Enfermagem, [S.L.], v. 69, n. 6, p. 1179-1187, dez. 2016. FapUNIFESP (SciELO). http://dx.doi.org/10.1590/0034-7167-2016-0364.

CORCINO, Cícero Oliveira et al. Avaliação do efeito do uso de agrotóxicos sobre a saúde de trabalhadores rurais da fruticultura irrigada. Ciência \& Saúde Coletiva, [S.L.], v. 24, n. 8, p. 3117-3128, ago. 2019. FapUNIFESP (SciELO). http://dx.doi.org/10.1590/141381232018248.14422017. 
FENZKE, Michele Nunes; MELLO, Marlise Capa Verde Almeida de; SANTOS, Kendra Natasha Sousa Castanha dos; CEZAR-VAZ, Marta Regina. Adoecimentos e fatores relacionados à saúde do trabalhador rural. Revista de Enfermagem Ufpe On Line, [s.l.], v.12, n. 8, p. 2214, 4 ago. 2018. Revista de Enfermagem, UFPE Online. http://dx.doi.org/10.5205/1981-8963v12i8a231532p2214-2226-2018.

FOLGADO, Cleber Adriano Rodrigues. Agrotóxicos: um problema invisibilizado. Brasília: Movimento dos Pequenos Produtores, 34 p, 2014.

LONDRES, Flávia. AGROTÓXICOS NO BRASIL: um guia para ação em defesa da vida. Rio de Janeiro: As-Pta - Assessoria e Serviços A Projetos em Agricultura Alternativa, 2011.Disponível em: https://br.boell.org/pt-br/2011/10/31/agrotoxicos-no-brasil-um-guia-para- acao-em-defesada-vida-0. Acesso em: 20 jan. 2021.

MORIN, Pâmela Vione; STUMM, Eniva Miladi Fernandes. Transtornos mentais comuns em agricultores, relação com agrotóxicos, sintomas físicos e doenças preexistentes. Psico, [S.L.],v. 49, n. 2, p. 196-205, 24 ago. 2018. EDIPUCRS. http://dx.doi.org/10.15448/19808623.2018.2.26814.

Disponível

em:

https://revistaseletronicas.pucrs.br/ojs/index.php/revistapsico/article/view/26814\#: :text=Res ultados $\% 3$ A $\% 20173 \% 20(47 \% 2$ C9,com $\% 20$ os $\% 20$ transtornos $\% 20$ mentais $\% 20$ comuns. Acesso em: 10 jan. 2021.

RISTOW, Letiane Peccin; BATTISTI, lara Denise Endruweit; STUMM, Eniva Miladi Fernandes; MONTAGNER, Sandra Emilia Drews. Fatores relacionados à saúde ocupacionalde agricultores expostos a agrotóxicos. Saúde e Sociedade, [S.L.], v. 29, n. 2, p. 1-11, 2020. FapUNIFESP (SciELO). http://dx.doi.org/10.1590/s0104-12902020180984.

SANTANA, Claudiana Mangabeira et al. Exposição ocupacional de trabalhadores rurais a agrotóxicos. Cadernos Saúde Coletiva, [S.L.], v. 24, n. 3, p. 301-307, set. 2016.

FapUNIFESP (SciELO). http://dx.doi.org/10.1590/1414-462x201600030199. SOUZA, Marcela Tavares de; SILVA, Michelly Dias da; CARVALHO, Rachel de. Revisão integrativa: o que é e como fazer. Einstein, São Paulo, p. 102-106, jan. 2010.

VIERO, Cibelle Mello; CAMPONOGARA, Silviamar; CEZAR-VAZ, Marta Regina; COSTA, Valdecir Zavarese da; BECK, Carmem Lúcia Colomé. Sociedade de risco: o uso dosagrotóxicos e implicações na saúde do trabalhador rural. Escola Anna Nery - Revista de Enfermagem, [S.L.], v. 20, n. 1, p. 99-105, 2016. FapUNIFESP (SciELO). http://dx.doi.org/10.5935/14148145.20160014. 